\title{
Condición ambiental del sedimento del sector oriental del golfo de Cariaco
}

\section{Environmental condition of sediment in the eastern sector of Cariaco Gulf}

\author{
María Valentina Fuentes Hernández ${ }^{1 *}$
}

\begin{abstract}
RESUMEN
En 2008, se evaluó la condición ambiental del sedimento de la región oriental del golfo de Cariaco (Venezuela) al relacionar las concentraciones químicas de metales traza e hidrocarburos policíclicos aromáticos (HAP) con las respuestas a pruebas toxicológicas estandarizadas. Para ello, en abril, los metales particionados en el sedimento y en el agua retenida fueron extraídos y medidos con ICP-OES. Los HAP fueron separados del sedimento con ultrasonido y medidos con HPLC-fluorescencia. Además, se ejecutaron pruebas de supervivencia de Artemia y actividad hemolítica. Las concentraciones de metales totales ( $\left.\mu \mathrm{g} \mathrm{g}^{-1}\right)$ fueron: $\mathrm{Cu}$ (29-91), $\mathrm{Cd}$ (1.9-4.9), $\mathrm{Cr}$ (4-36), $\mathrm{Ni}$ (10-33), $\mathrm{Pb}$ (3-9) y $\mathrm{Zn}$ (32-112). En el agua fueron detectados, en $\mu \mathrm{g} \mathrm{L}^{-1}$ : $\mathrm{Pb}(33-$ 56) y $\mathrm{Cr}$ (154-237). Las concentraciones de HAP (3-5 anillos) fueron bajas, con predominio de fluoranteno (22-71 $\mathrm{ng} \mathrm{g}^{-1}$ ), en particular, pirolítico. Solamente, en algunas localidades, las concentraciones de $\mathrm{Cu}$, $\mathrm{Ni}$ y $\mathrm{Cd}$ del sedimento superaron los criterios de calidad. No hubo mortandad de larvas durante las pruebas toxicológicas y la actividad hemolítica fue menor que $2 \%$. Una vez realizado el contraste se demuestra que los sedimentos tienen alta calidad ecológica.
\end{abstract}

Palabras clave: Agua, riesgo ambiental, sedimento, tóxicos, Venezuela

\begin{abstract}
In 2008, the environmental status of sediment in the eastern region of the Gulf of Cariaco (Venezuela) was evaluated by comparing chemical concentrations of trace metals and polycyclic aromatic hydrocarbons (PAHs) to responses to standardized toxicological tests. In April, the metals partitioned in the sediment and retained water were extracted and measured using ICPOES analysis. The PAHs were separated from the sediment using ultrasound and measured with HPLC-fluorescence detectors. In addition, Artemia survival was evaluated and hemolytic activity tests were performed. The total metal concentrations $\left(\mu \mathrm{g} \mathrm{g}^{-1}\right)$ were: $\mathrm{Cu}$ (29-91), $\mathrm{Cd}(1.9-$ 4.9), $\mathrm{Cr}$ (4-36), Ni (10-33), $\mathrm{Pb}(3-9)$ and $\mathrm{Zn}$ (32-112). Results of the analysis of water ( $\left.\mu \mathrm{g} \mathrm{L}^{-1}\right)$ were $\mathrm{Pb}$ (33-56), $\mathrm{Cr}$ and (154-237). The concentrations of PAHs (3-5 rings) were low, with a
\end{abstract}

1 Escuela de Ciencias, Núcleo de Sucre, Universidad de Oriente, Cerro Colorado, Cumaná, Venezuela. ORCID: https://orcid.org/0000-0002-7705-3368,mfuentes@udo.edu.ve; mvfuentesh@gmail.com* 
predominance of fluoranthene (22-71 $\left.\mathrm{ng} \mathrm{g}^{-1}\right)$, mainly pyrolytic. Concentrations of $\mathrm{Cu}, \mathrm{Ni}$ and $\mathrm{Cd}$ in the sediment exceeded quality criteria in only a few locations. There was no mortality of larvae during toxicological tests and hemolytic activity was less than $2 \%$. These results show that the sediments analyzed have high ecological quality.

Keywords: Sediment, water, toxins, environmental risk, Venezuela

\section{INTRODUCCIÓN}

Muchos contaminantes ambientales orgánicos e inorgánicos pueden ser introducidos a las aguas superficiales costeras de manera natural o antrópica, donde pueden estar disueltos o ser adsorbidos en las partículas finas de limo, arcilla o materia orgánica transportadas al mar por el viento, corrientes de agua dulce y las mareas. Sin importar su procedencia, estas sustancias son acumuladas en los sedimentos marinos de áreas de baja energía cinética, como ensenadas, bahías, lagunas, golfos, etc., y distribuidas, de acuerdo con su afinidad, a un compartimiento particular del sedimento, en donde las condiciones fisicoquímicas pueden favorecer su adsorción o desorción, y determinar su biodisponibilidad (Carballo et al. 2010). De hecho, siempre existe una especie química controladora del acopio, sin ser la causante de las altas concentraciones. Verbigracia, un sedimento rico en sulfuros retiene metales o de modo similar, la materia orgánica puede favorecer la acumulación de hidrocarburos policíclicos aromáticos (Araujo et al. 2006). Empero, las variaciones ambientales pueden provocar la liberación de estas sustancias acumulativas y tóxicas adheridas a las partículas del sedimento hacia el agua, donde son más disponibles química y biológicamente (Fuentes, 2010). En específico, cuando estas especies químicas cautivas están presentes en cantidades superiores a una concentración umbral pueden causar efectos tóxicos adversos a los organismos vivos e impactar indirectamente la salud humana, por ello cualquier contaminante que ingrese al medio marino afecta, de forma potencial, la ecología (Khaled et al. 2017; Fuentes, 2010).

Como el sedimento es un componente importante de los ecosistemas marinos y provee hábitats que sostienen una gran variedad de organismos bentónicos, la presencia de metales e hidrocarburos policíclicos aromáticos representan riesgos potenciales para su bienestar (Simpson et al. 2005; Swarnalatha \& Nair, 2017). En tal sentido, las evaluaciones ecotoxicológicas consideran muy importante conocer las consecuencias biológicas que estos puedan provocar a los organismos (Carballo et al. 2010). 
Tomando en cuenta estas consideraciones, una buena aproximación para realizar una evaluación efectiva de la condición ambiental de un sedimento requiere contrastar las concentraciones de los compuestos químicos asociados a él, que miden la contaminación con los resultados de pruebas toxicológicas en el laboratorio y la ocurrencia de efectos biológicos adversos en condiciones estandarizadas. Los resultados permiten categorizar la condición ambiental desde no degradada (sin ningún parámetro alterado) hasta degradado (todos los parámetros alterados), tomando en consideración las guías de calidad para especies químicas y los resultados de las pruebas toxicológicas que cubren distintos estadios de vida (Chapman, 1996; Long et al. 2002).

El golfo de Cariaco es una de las áreas pesqueras más importantes de la región nororiental de Venezuela. En su región más oriental está ubicado el Saco, cuyos sedimentos acumulan especies químicas naturales y antropogénicas, debido a la contribución de múltiples factores como la meteorización natural de rocas sedimentarias de la Formación Frontada, la geomorfología que condiciona su baja energía hidrodinámica, el drenaje continental de material fino desde zonas bajas y pantanosas que caen por la costa sur y el extremo oriental arrastrado por el río Cariaco y quebradas, la presencia de grandes formaciones de manglares, los cuales junto a la elevada producción planctónica y los procesos de afloramiento aportan y renuevan una gran cantidad de materia orgánica particulada (Caraballo, 1982; Fuentes et al. 2010). Además, recibe directamente aguas servidas y desechos sólidos de las poblaciones circundantes con carencia entre $81 \%$ y $96 \%$ de servicios cloacales (Quintero et al. 2002) y restos de pesticidas, herbicidas y abonos de la extensa actividad agrícola realizada en una región aledaña a Cariaco (Martínez, 2002). Una contribución adicional es la liberación a la atmósfera de residuos de combustibles del tráfico rodante y flotante que pueden depositarse en el ecosistema. Estas condiciones logran modificar la calidad natural de este ecosistema, con gran importancia ecológica, socioeconómica y científica, y disminuir, de forma notable, los recursos vivos (Fuentes, 2010). Aunque han transcurrido 13 años de la obtención y procesamiento de esta data, ella complementa la historia de la región, en concreto, porque congrega información simultánea sobre concentración de las especies químicas señaladas y pruebas toxicológicas en esta relevante área marina, especialmente cuando el Instituto Oceanográfico de Venezuela, custodio de esta información, ha sido desvalijado y quemado.

Por ello, el propósito de este trabajo es establecer, con certeza, la 
condición ambiental respecto a la presencia de metales e hidrocarburos policíclicos aromáticos de los sedimentos de la región oriental del golfo de Cariaco y su potencial efecto sobre los organismos, lo cual proporcionará datos importantes para planificar el desarrollo sustentable de la zona, el cual obliga a su conservación y uso racional.

\section{MATERIALES Y MÉTODOS}

El saco del golfo de Cariaco está enclavado en la zona oriental de la península de Araya (Venezuela), aproximadamente entre $63^{\circ} 38^{\prime}$ y $63^{\circ} 46^{\prime}$ de longitud oeste y $10^{\circ} 28^{\prime}$ y $10^{\circ} 32^{\prime}$ de latitud norte (Fig.1). Su extensión es $29 \mathrm{~km}^{2}$, profundidad máxima $35 \mathrm{~m} \mathrm{y}$ salinidad media 35 UPS. Clima semidesértico, precipitación anual menor a $250 \mathrm{~mm}$, temperaturas entre $26^{\circ} \mathrm{C} \mathrm{y}$ $27^{\circ} \mathrm{C}$, moderadas por los vientos alisios NE (2-5 $\left.\mathrm{m} \mathrm{s}^{-1}\right)$. La evaporación es superior a $2000 \mathrm{~mm}$ año-1. Su hidrología está poco influenciada por ríos, quebradas y escorrentías (Caraballo, 1982; Quintero et al. 2005).

Como toda zona resguardada, la energía del agua es baja y las partículas se transportan preeminentemente suspendidas, lo cual justifica la presencia de sedimentos mal clasificados con detritos de rocas sedimentarias, esqueletos, conchas de moluscos, arrecifes marginales, caparazones de foraminíferos planctónicos y bentónicos, fracción limosa y arcilla procedentes de suelos adyacentes y arrastradas por el río Cariaco (Caraballo, 1982; Quintero et al. 2006).

El saco del golfo colinda con los municipios Mejías y Ribero, cuya población aproximada es de 90884 habitantes (INE, 2014) y sus actividades económicas principales son la agricultura y la pesquería (Quintero et al. 2002). Los residuos de estos núcleos poblados, suelos cultivados, tráfico automotor y quema de vegetación introducen al ecosistema sustancias químicas nocivas.

Para recoger las muestras de los sedimentos superficiales, en abril 2008, temporada seca, se establecieron doce estaciones a diferentes profundidades (Ests.), ubicadas con un sistema de posicionamiento global diferencial (Cuadro 1, Fig. 1). Previo al muestreo, todo el equipo fue lavado con $\mathrm{HNO}_{3}$ $10 \%$, enjuagado con agua Millipore y finalmente, con agua de mar de la estación en cuestión. Las muestras fueron recogidas con una draga Diez Laffont de $0.02 \mathrm{~m}^{2}$ de área, a una profundidad aproximada de $5 \mathrm{~cm}$, y conservadas a $4^{\circ} \mathrm{C}$ hasta su análisis, dos días después. 


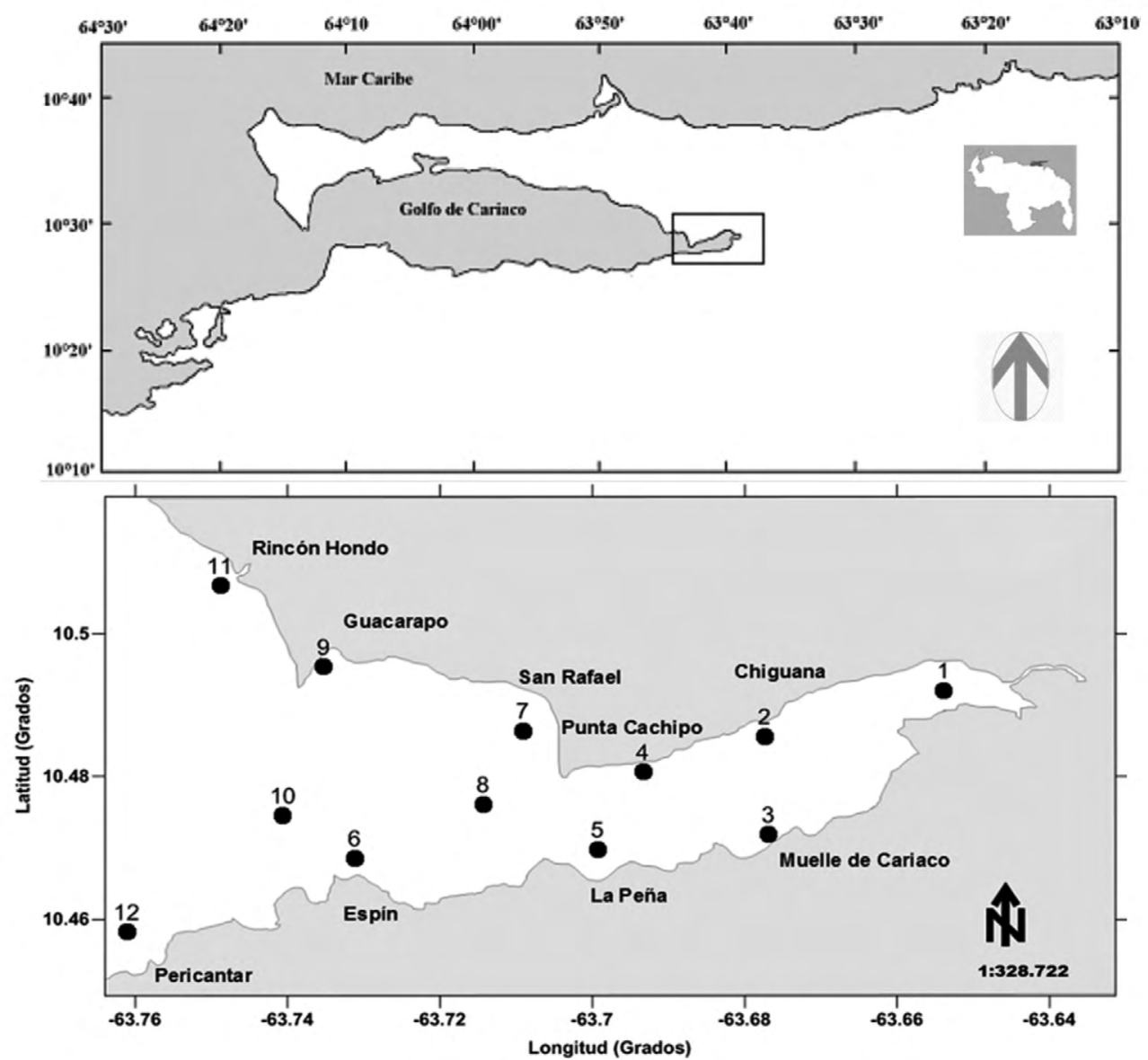

Fig. 1. Saco del golfo de Cariaco. Localización geográfica. Estaciones de muestreo (Fuentes-Hernández et al. 2019)

Fig. 1. Eastern region of the Gulf of Cariaco. Geographic location. Sampling stations (Fuentes-Hernández et al. 2019)

La fracción gruesa del sedimento $\operatorname{seco}(>2 \mathrm{~mm})$ fue separada por tamización en un equipo Ro-Tap con una columna Tyler; mientras que las fracciones menores, limo y arcilla, por sedimentación, con el método del hidrómetro densímetro (Bouyoucos, 1962). Para ello, la muestra se mezcló con polifosfato de sodio y agua destilada, agitó, homogeneizó y procesó como está descrito en Primo \& Carrasco (1973). Una vez obtenidos los porcentajes de arena, limo y arcilla se determinó la textura sedimentaria, mediante el triángulo de Shepard (1954). 
Cuadro 1. Coordenadas geográficas de las estaciones de muestreo en el saco del golfo de Cariaco

Table 1. Geographical coordinates of the sampling stations in the eastern region of the Gulf of Cariaco

\begin{tabular}{llll}
\hline $\begin{array}{c}\text { Esta- } \\
\text { ción }\end{array}$ & Latitud norte & Longitud oeste & $\begin{array}{c}\text { Profun- } \\
\text { didad } \\
(\mathrm{m})\end{array}$ \\
\hline 1 & $10^{\circ} 29,313^{\prime}$ & $63^{\circ} 39,140^{\prime}$ & 3.5 \\
2 & $10^{\circ} 29,186^{\prime}$ & $63^{\circ} 40,331^{\prime}$, & 3.0 \\
3 & $10^{\circ} 28,180^{\prime}$ & $63^{\circ} 39,140^{\prime}$ & 6.0 \\
4 & $10^{\circ} 28,510^{\prime}$ & $63^{\circ} 41,360^{\prime}$ & 11.0 \\
5 & $10^{\circ} 28,309^{\prime}$ & $63^{\circ} 41,388^{\prime}$ & 11.2 \\
6 & $10^{\circ} 27,994^{\prime}$ & $63^{\circ} 43,615^{\prime}$ & 19.0 \\
7 & $10^{\circ} 29,109^{\prime}$ & $63^{\circ} 42,327^{\prime}$ & 1.3 \\
8 & $10^{\circ} 28,432^{\prime}$ & $63^{\circ} 42,859^{\prime}$ & 18.0 \\
9 & $10^{\circ} 29,720^{\prime}$ & $63^{\circ} 44,112^{\prime}$ & 20.5 \\
10 & $10^{\circ} 38,241^{\prime}$ & $63^{\circ} 44,558^{\prime}$ & 30.0 \\
11 & $10^{\circ} 30,241^{\prime}$ & $63^{\circ} 44,558^{\prime}$ & 9.5 \\
12 & $10^{\circ} 27,047^{\prime}$ & $63^{\circ} 46,478^{\prime}$ & 34.0 \\
\hline
\end{tabular}

El carbono orgánico (Corg.) se determinó mezclando $0.5 \mathrm{~g}$ del sedimento tamizado con diámetro menor que $2 \mathrm{~mm}$, con $10 \mathrm{~mL}$ de $0.5 \mathrm{~mol} \mathrm{~L}^{-1}$ de $\mathrm{K}_{2} \mathrm{Cr}_{2} \mathrm{O}_{7}$ y $20 \mathrm{~mL}$ de ácido sulfúrico concentrado. El calor de la reacción liberada es suficiente para provocar la combustión húmeda del Corg. El dicromato de potasio excedente es titulado con una solución estándar $0.2 \mathrm{~mol}$. $\mathrm{L}^{-1}$ de $\mathrm{Fe}\left(\mathrm{NH}_{4}\right)_{2}\left(\mathrm{SO}_{4}\right)_{2}$, en presencia de sulfato de plata para corregir el error positivo debido al cloruro (Gaudette et al. 1974).
Los carbonatos $\left(\mathrm{CaCO}_{3}\right)$ se obtuvieron por termogravimetría a $800^{\circ} \mathrm{C}$. $1 \mathrm{~g}$ de sedimento tamizado, menor que $2 \mathrm{~mm}$, fue colocado en una mufla por períodos de $2 \mathrm{~h}$ a cada temperatura del gradiente $105,300,550$ y $800^{\circ} \mathrm{C}$. La cantidad de carbonato se calculó con la diferencia de masas obtenidas a 550 y $800^{\circ} \mathrm{C}$.

Los metales totales fueron determinados, por triplicado, de la siguiente manera: $1 \mathrm{~g}$ de sedimento seco, procedente de la fracción granulométrica menor que $2 \mathrm{~mm}$, después de tamizado, se digirió durante $12 \mathrm{~h}$ en $10 \mathrm{~mL}$ de ácido nítrico concentrado, luego se calentó durante $2 \mathrm{~h}$ a $80^{\circ} \mathrm{C}$ y por $2 \mathrm{~h}$ más a $120^{\circ} \mathrm{C}$ (Rojas et al. 2005). Al enfriar, se filtró y enrasó, con agua ultrapura, hasta $25 \mathrm{~mL}$. Todas las muestras se midieron por espectrometría de emisión óptica (OES) con un plasma inductivamente acoplado (ICP), ejecutada con un equipo Perkin Elmer Optima 5300 DV. La adecuación del método para el requerimiento indicado fue validada con un material de referencia certificado de sedimentos marinos (HISS-1). La veracidad resultó mayor que $92 \%$, la precisión entre $0 \%$ y 3\%, la exactitud superior a 93\%. Los límites de detección obtenidos en la validación, en $\mu \mathrm{g}$ $\mathrm{g}^{-1}$, fueron $\mathrm{Cd}$ (0.30), $\mathrm{Cu}$ (1), Cr (0.05), $\mathrm{Mn}$ (1), Pb (0.3), Ni (0.2), Zn (1).

El agua retenida en el sedimento fue sustraída por centrifugación refrigerada $\left(20^{\circ} \mathrm{C}\right)$, a $2330 \mathrm{rpm}$, filtrada 
por papel Whatman $42 \mu \mathrm{m}$. Los metales totales presentes en el agua fueron cuantificados, por triplicado, mediante espectrometría de emisión óptica con un plasma inductivamente acoplado (OES-ICP). Las soluciones estándares fueron preparadas en agua de mar artificial para minimizar el efecto matriz. Para validar el método, se determinaron los límites de detección $\left(\mu \mathrm{g} \mathrm{L}^{-1}\right)$ : $\mathrm{Cd}$ (10), $\mathrm{Cu}$ (30), Pb (10), Ni (6), Zn (50), Cr (41). La precisión de los resultados fue menor de $2 \%$.

Los hidrocarburos aromáticos policíclicos (HAP) fueron extraídos mediante ultrasonido con la metodología descrita en Banjoo \& Nelson (2005). El reactivo extractante fue diclorometano, seguido de purificación del extracto con hexano-diclorometano en una columna cromatográfica miniatura con virutas de cobre activado para remover el azufre elemental A continuación, la separación de sus componentes se realizó con un cromatógrafo líquido de alta eficacia (HP 1 100) con detector de fluorescencia y una columna LiChrospher®PAH (250-3-HPLC), bajo las condiciones descritas por Fuentes et al. (2012).

Para la identificación y cuantificación de HAP se utilizó una mezcla SRM 1647e del Instituto Nacional de Estándares y Tecnologías (NIST) y patrones individuales. La veracidad del método fue superior a $92 \%$, exactitud más de $90 \%$, la precisión menor que
3\%. Los límites de detección, en ng $\mathrm{g}^{-1}$, fueron: naftaleno (0.04), fenantreno $(0.01)$, antraceno $(0.011)$, fluoranteno (0.002), pireno (0.05).

El cálculo del coeficiente de correlación de Pearson permitió medir la relación estadística lineal entre las variables aleatorias estudiadas.

La evaluación de la toxicidad aguda se realizó, en el agua extraída del sedimento con la prueba de supervivencia Artemia de la norma mexicana NMX-AA-110-1995-SCFI (SCFI, 1995). Una vez eclosionados los nauplios de Artemia sp., 10 de ellos, se colocaron en $2 \mathrm{~mL}$ de diluciones del agua con agua de mar artificial (25$100 \%$ ), durante 24 horas a temperatura ambiente. Posteriormente, se cuentan los individuos muertos y vivos. El criterio de muerte fue la inmovilidad de los apéndices y del tracto digestivo durante un período de observación de 10 segundos con una lupa binocular.

Para averiguar la probabilidad tóxica de las sustancias presentes en los extractos orgánicos del sedimento se modificó la prueba supervivencia de Artemia franciscana (SCFI 1995). El proceso de extracción fue descrito por Banjoo \& Nelson (2005) para HAP. Las soluciones de prueba fueron diluciones del extracto con agua de mar artificial (1:4, 1:8, 1:16). La exposición de los organismos siguió el protocolo descrito, previamente, para el agua extraída del sedimento. Simultáneamente, para 
evaluar $\mathrm{LC}_{50}$ fueron realizados ensayos con diluciones acuosas de $\mathrm{ZnSO}_{4}$ $\left(\mathrm{LC}_{50} 35 \mathrm{mg} \mathrm{L}^{-1}\right)$, naftaleno en DMS $5 \% \mathrm{v} / \mathrm{v}\left(\mathrm{LC} 50600 \mu \mathrm{g} \mathrm{L}^{-1}\right)$ y DMS 5\% $\mathrm{v} / \mathrm{v}$ (control negativo).

La prueba de eritrocateresis por acción del agua retenida en los sedimentos fue realizada con el protocolo propuesto por Key et al. (2002), el cual consiste en incubar durante 90 minutos, en un baño de agua a $37^{\circ} \mathrm{C}$, diluciones (25-100\%) del agua extraída del sedimento con sangre humana $\left(\mathrm{ORh}^{+}\right)$. La medición de la oxihemoglobina liberada se realizó con un espectrómetro de absorción molecular UV/VIS Jenway 6300 a $540 \mathrm{~nm}$., y debe ser directamente proporcional al daño provocado, al ser expuestos a sustancias nocivas.

Para valorar la capacidad potencial de estas sustancias químicas para provocar problemas de salubridad ambiental que puedan afectar a la flora, fauna o salud humana fue elaborado un arreglo bidimensional, emulando la matriz de decisión tabulada para una evaluación semicuantitativa de sedimentos reseñada en Simpson et al. (2005), donde se compilaron las caracterizaciones química y toxicológica, al atender a la ponderación relativa indicada en el cuadro 2. Para pronosticar posibles efectos químicos se usaron dos conjuntos de concentraciones como valores críticos: los Intervalo de Efectos Bajos (ERL) y Efectos Medios (ERM) para sedimentos y los Criterios de Concentración Continua (CCC) y Concentración Máxima (CCM) para proteger la vida acuática marina, ambos emitidos por la Administración Oceánica y Atmosférica (NOOA). Respecto a esto, Long \& Morgan (1990) y O'Connor \& Paul (2004) señalan que ERL y ERM identifican a los percentiles 10 y 50 de un conjunto de concentraciones que coocurren con la toxicidad: De hecho, ERL es aquella concentración de una especie química en el sedimento, por debajo de la cual, los efectos adversos son rara vez observados $(<10 \%)$, concentraciones iguales o superiores a ERL pero inferiores a ERM representan un intervalo de posibles efectos (10-50\%). Por otro lado, CCC es una estimación de la concentración más alta de una sustancia, en el medio acuático, a la que pueda ser expuesta, indefinidamente, la comunidad acuática, sin que se produzcan consecuencias desfavorables (crecimiento, reproducción, supervivencia) y por CCM a la concentración más alta de una especie química en la columna de agua que puede un organismo acuático ser expuesto de manera aguda sin causar un efecto adverso $\left(\mathrm{LC}_{50}\right)(\mathrm{Bu}-$ chman, 2008). La valoración relativa conlleva a la total (Cuadro 2), de tal manera que un sedimento de "Alta calidad" coincidirá con la ponderación $\mathbf{1}$, "Calidad media" con la clasificación 2 y, "Baja calidad” corresponderá 3. 


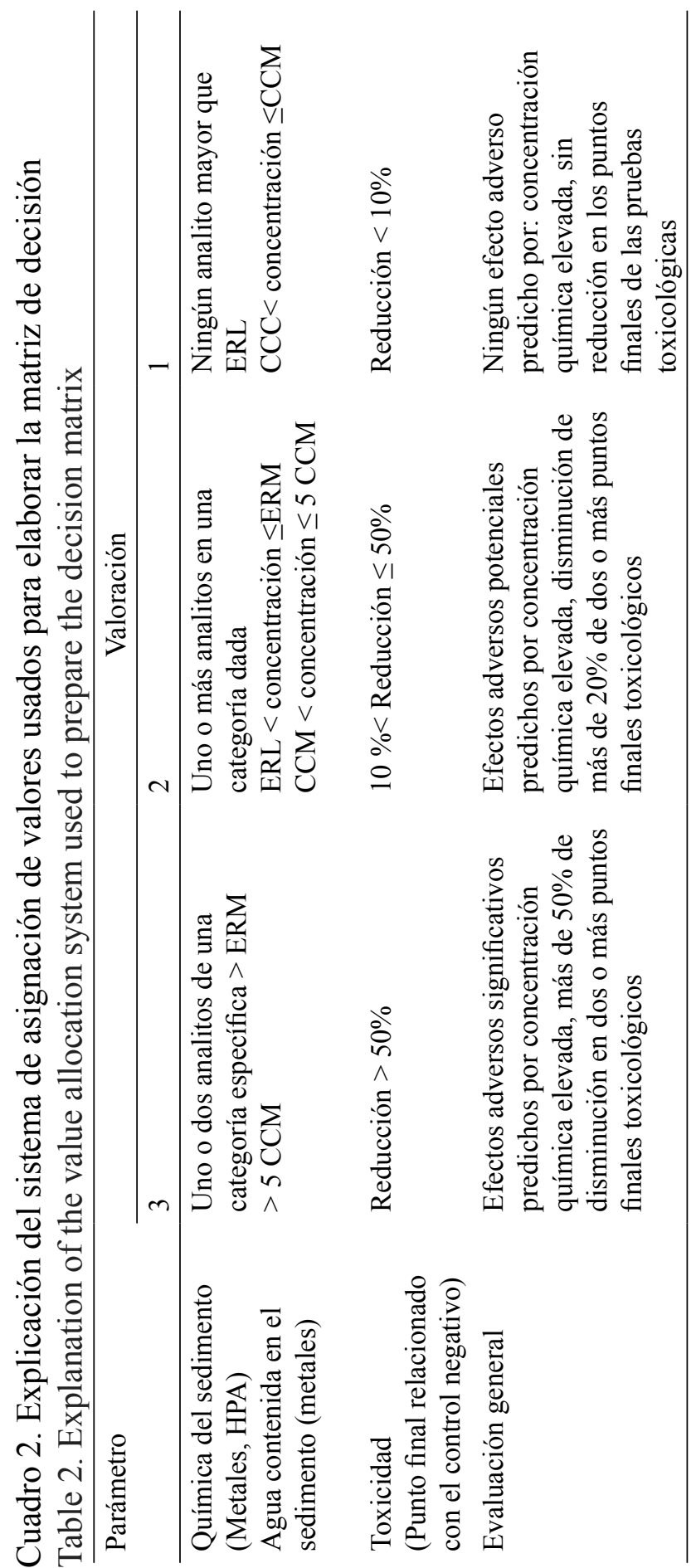

\section{RESULTADOS}

En los estudios medioambientales, de forma común, el conjunto de magnitudes medidas muestran una dispersión o desviación grande respecto a la media. Ante esta heterogeneidad, el valor promedio no tiene méritos. No obstante, cuando los datos son homogéneos, la media aritmética es altamente significativa (Gutiérrez, 2012), por ello en este trabajo, solo los resultados homogéneos serán representados por la media y la desviación estándar.

La textura predominante en los sedimentos del ecosistema fue arenolimosa (Ests. 1, 3, 6, 7, 8, 10), con porcentajes comparables de arena y limo (Cuadro 3), situación similar presentó la textura limoarenosa (Ests. $4,5)$, mientras que la textura arenosa se presentó en la zona más occidental (Ests. $9,11,12)$ y frente a Chiguana (Est. 2). Este conjunto caracteriza al ecosistema como predominantemente arenolimoso. 
Cuadro 3. Composición textural, carbono orgánico y carbonatos (\%) en los sedimentos del saco del golfo de Cariaco

Table 3. Textural composition, organic carbon and carbonates (\%) in the sediments from the eastern region of the Gulf of Cariaco

\begin{tabular}{lccclcc}
\hline Est. & Arena & Limo & Arcilla & Textura & Corg & Carbonatos \\
\hline 1 & 68.2 & 23.3 & 8.5 & Arena limosa & 1.3 & 3.0 \\
2 & 85.5 & 8.7 & 5.8 & Arena & 1.2 & 2.7 \\
3 & 50.2 & 46.0 & 3.8 & Arena limosa & 4.2 & 2.8 \\
4 & 44.2 & 50.0 & 5.8 & Limo arenoso & 4.1 & 3.5 \\
5 & 46.2 & 48.0 & 5.8 & Limo arenoso & 2.8 & 2.6 \\
6 & 74.2 & 20.0 & 5.8 & Arena limosa & 1.3 & 0.7 \\
7 & 49.0 & 44.0 & 7.0 & Arena limosa & 4.1 & 4.4 \\
8 & 49.0 & 44.0 & 7.0 & Arena limosa & 4.7 & 3.9 \\
9 & 90.2 & 6.0 & 3.8 & Arena & 0.5 & 0.7 \\
10 & 56.2 & 39.3 & 4.5 & Arena limosa & 1.0 & 0.8 \\
11 & 88.2 & 7.3 & 4.5 & Arena & 0.1 & 0.7 \\
12 & 90.2 & 6.0 & 3.8 & Arena & 0.4 & 1.0 \\
\hline
\end{tabular}

Directamente relacionado con limo ( $\mathrm{r}=0.86, P<0.05)$, pero inverso a la arena $(\mathrm{r}=-0.86, P<0.05)$ se mostró el Corg., cuyas concentraciones variaron entre 0.1 y $4.7 \%$. Las zonas más ricas fueron: centro del ecosistema (Est. 8), muelle de Cariaco (Est. 3), punta Cachipo (Est. 4) y San Rafael (Est. 7). En las restantes localidades, los contenidos fueron menores que $2.8 \%$.

De manera similar a Corg., se acumuló $\mathrm{CaCO}_{3}(\mathrm{r}=0.86, P<0.05)$ en el oriente (Ests. 1-5), norte (Est. 7) y centro (Est. 8), con valores comprendidos entre $2.6 \%$ (Est. 5) y $4.4 \%$, (Est.
7). En el resto del ecosistema varió entre $0.7 \%$ (Est. 11) y $0.9 \%$ (Est. 12). Además, dicho parámetro correlacionó con arena $(\mathrm{r}=-0.70, P<0.05)$, limo $\mathrm{y}$ arcilla $(\mathrm{r}=0.66, P<0.05)$.

Las concentraciones totales de Cd (1.9-4.9 $\left.\mu \mathrm{g} \mathrm{g} \mathrm{g}^{-1}\right), \mathrm{Cr}\left(4-36 \mu \mathrm{g} \mathrm{g}^{-1}\right)$, $\mathrm{Cu}\left(29-91 \mu \mathrm{g} \mathrm{g}^{-1}\right)$, Mn (12-63 $\left.\mu \mathrm{g} \mathrm{g}^{-1}\right)$, $\mathrm{Pb}\left(3-9 \mu \mathrm{g} \mathrm{g}^{-1}\right)$, Ni (10-43 $\left.\mu \mathrm{g} \mathrm{g}^{-1}\right)$ y $\mathrm{Zn}$ (72-113 $\mu \mathrm{g} \mathrm{g}^{-1}$ ) (Cuadro 4) en el sedimento mostraron mayor acumulación hacia el oriente (Ests. 1-3), costa norte (Ests. 4,7$)$ y centro (Est. 8) y sus menores contenido en la costa sur (Ests. 5, 6, 12), correlacionadas con Corg. 
$(\mathrm{r}=0.51, P<0.05), \mathrm{CaCO}_{3}(0.57<\mathrm{r}$ $<0.89, P<0.05)$, limo ( $\mathrm{r}<0.63, P$ $<0.05)$ y arcilla $(\mathrm{r}<0.64, P<0.05)$. También se encontró una significativa afinidad Mn-metal ( $\mathrm{r}>0.50, P<0.05)$, $\mathrm{Zn}-$ metal $(\mathrm{r}>0.64)$ y entre $\mathrm{Cd}, \mathrm{Cu}, \mathrm{Ni}$ y $\mathrm{Pb}(\mathrm{r}>0.60, P<0.05)$.

En el agua extraída del sedimento, los únicos metales presentes fueron $\mathrm{Pb}$ y $\mathrm{Cr}$. $\mathrm{Pb}$ con concentraciones comprendidas entre $32 \mu \mathrm{g} \mathrm{L}^{-1}$ (Est. 8) y 55 $\mu \mathrm{g} \mathrm{L}^{-1}$ (Est. 9), $47 \pm 6 \mu \mathrm{g} \mathrm{L}^{-1}$. Cr entre $154 \mu \mathrm{g} \mathrm{L}^{-1}$ (Est. 11) y $237 \mu \mathrm{g} \mathrm{L}^{-1}$ (Est. 12), $191 \pm 27 \mu \mathrm{g} \mathrm{L}^{-1}$. Esta distribución fue casi homogénea en el área. $\mathrm{Cd}$ no fue detectado, mientras que $\mathrm{Cu}, \mathrm{Ni}$ y $\mathrm{Zn}$ mostraron valores por debajo del límite de detección.

En los sedimentos superficiales del saco del golfo de Cariaco, los hidrocarburos aromáticos policíclicos de alta masa molar (HAPA) fueron los más abundantes, representados por fluoranteno, pireno y benzo[b] fluoranteno (Cuadro 4). Su sumatoria varió entre $23.4 \mathrm{ng} \mathrm{g}^{-1}$ (Est. 11, Rincón Hondo) y $72.8 \mathrm{ng} \mathrm{g}^{-1}$ (Est. 4, punta Cachipo), fuertemente influenciada por la concentración del fluoranteno (22-71 $\left.\mathrm{ng} \mathrm{g}^{-1}\right)$; mientras que, la totalidad de las concentraciones de los hidrocarburos aromáticos policíclicos de baja masa molar (HPAB), cuyos únicos representantes fueron naftaleno, antraceno y fenantreno, variaron entre $3.7 \mathrm{ng} \mathrm{g}^{-1}$ (Est. 12, Pericantar) y 17.3 ng $\mathrm{g}^{-1}$ (Est. 8, centro). La mayoría de los HAP se acumularon en muelle de
Cariaco (Est. 3), punta Cachipo (Est. 4), San Rafael (Est.7), Guacarapo (Est. 9) y centro-occidente (Est. 10), sin afinidad significativa con otros parámetros investigados $(0.40, P<0.05)$.

No hubo mortalidad de los nauplios de Artemia sp., después de su exposición a las diluciones del agua sustraída y del extracto orgánico del sedimento. Por otra parte, la lisis de los glóbulos rojos al estar en contacto con el agua extraída fue insignificante, menor que $2 \%$.

\section{DISCUSIÓN}

En los sedimentos superficiales del saco del golfo de Cariaco, la distribución del tamaño de grano es variable pero controlada, en particular, por la suspensión (Quintero et al. 2006). En las áreas más tranquilas, las arenas medias, finas y muy finas están presentes en proporciones comparables con los limos, provenientes de la materia orgánica descompuesta. Por ello, predominan los sedimentos arenolimosos, excepto en punta Cachipo y La Peña (limoarenosos) y en la costa norte (arenas medias bioclásticas).

Los porcentajes de carbono orgánico comprendido entre $0.3 \% \mathrm{y}$ 4.7\% indican una importante deposición orgánica natural, incrementada por descargas domésticas (Salazar, 1991). Desde otro punto de vista, Ferráz-Reyes (1987) explicó que el saco del golfo de Cariaco tiene una alta 
productividad primaria. Al acumularse grandes cantidades de fitoplancton, la dinámica de las corrientes y la morfología de la zona proveen condiciones que favorecen la deposición de una cantidad considerable en el fondo marino, y provocan una importante acumulación de carbono orgánico.

Las concentraciones de los metales estuvieron por debajo de los patrones geoquímicos de la fracción limo-arcilla. Ello, aunado a su localización indican que la distribución de los metales está influenciada por la dinámica sedimentaria y el efecto de la contaminación es poco (Krupadam et al. 2006; Ahmad et al. 2009); es decir, los sedimentos son, a priori, materiales detríticos de las rocas sedimentarias de la Formación Frontado y de los aportes arrastrados, por los cursos de agua, desde la costa sur y oriente. De hecho, los sedimentos del área estudiada son residuos de la erosión de los suelos de la península de Araya y de los suelos húmicos aledaños al río Carinicuao (Quintero et al. 2006). Particularmente, los metales presentan mayor concentración en la zona más oriental, por la influencia de la materia orgánica terrestre acarreada por el río. Esta materia es distribuida entre las partículas minerales amorfas del sedimento, las cuales actúan como sitios de enlace para contaminantes (Simpson et al. 2005).

En las áreas costeras es muy común encontrar abundantes cantidades de Fe, Mn y Zn, procedentes de la corteza terrestre, que interactúan con otros metales, sin mostrar toxicidad (Rubio et al. 1995). Cuando ellos correlacionan significativamente con los demás metales es una fuerte evidencia que provienen de la misma fuente (García-Rico et al. 2004; Belzunce-Segarra et al. 2008). Por otro lado, MacDonald et al. (1991) resaltan que una certeza de la existencia de residuos antrópicos es la poca afinidad entre $\mathrm{Zn}$ y Mn con otros metales como $\mathrm{Cu}, \mathrm{Cd}$ y $\mathrm{Pb}$.

En toda el área de estudio, las concentraciones de $\mathrm{Cd}$ superaron al intervalo de efectos bajos (ERL), concentración por debajo de la cual, rara vez, ocurren efectos adversos (10\%, Cuadro 4). $\mathrm{Cu}$ mostró este mismo comportamiento en tres cuartas partes del ecosistema y $\mathrm{Ni}$ solamente en la mitad. Empero, todas fueron inferiores al intervalo de efectos medios (ERM), valor representativo de concentraciones por encima de la cual puedan ocurrir efectos adversos (50\%), ambos valores referenciales de la Administración Nacional Oceánica y Atmosférica (NOOA, 2019). No obstante, fueron similares a los contenidos informados en otras investigaciones (Martínez, 2002; 2016; Márquez et al. 2005; Velásquez, 2005). Esto indica que son sedimentos de calidad estándar, es decir, no provocan efectos biológicos adversos a los organismos, ni daños a la salud. Por ello, las fuentes adicionales de $\mathrm{Cd}, \mathrm{Ni}, \mathrm{Cu}, \mathrm{Pb}$ pueden estar 
relacionadas con la descomposición de fitoplancton depositado en el fondo, el lavado de suelos agrícolas, las descargas urbanas y la quema de vegetación y basura (García-Rico et al. 2004).

En cuanto a las concentraciones de los metales en el agua de mar, $\mathrm{Pb}$ superó $20 \mu \mathrm{g} \mathrm{L}^{-1}$, determinado por Nayar et al. (2004) en el estuario de Punggol (Singapur), pero fue menor que $106 \mu \mathrm{g} \mathrm{L}^{-1}$, informado por Man et al. (2004) en un humedal en Hong Kong, ambos ecosistemas catalogados como no contaminados. Por otro lado, fueron significativamente menores que 1 $000 \mu \mathrm{g} \mathrm{L}^{-1}$, concentración letal de $\mathrm{Pb}$ (LC50-96 h) para Chasmagmathus granulata en el estuario Bahía Blanca de Argentina (Ferrer et al. 2006) y menores que $140 \mu \mathrm{g} \mathrm{L}^{-1}$, que es el criterio de concentración máxima (CCM), no obstante, fueron mayores que el criterio de concentración continua (CCC, $\left.8.1 \mu \mathrm{g} \mathrm{L}^{-1}\right)$ referidos por la NOOA (Buchman, 2008). Este plomo parece ser dual, autóctono y antropogénico, inferido por su correlación con arena y $\mathrm{Cr}(\mathrm{r}=0.40, P<0.05)$, dado que sus mayores concentraciones están registradas cerca de centros poblados.

Las concentraciones de $\mathrm{Cr}$ en agua de mar fueron superiores a las informadas por Botté et al. (2007) para el estuario de Bahía Blanca en Argentina $\left(9.4 \mu \mathrm{g} \mathrm{L}^{-1}\right)$ y por Man et al. (2004) en Mai Po (121 $\left.\mu \mathrm{g} \mathrm{L}^{-1}\right)$, ambientes impolutos; no obstante, estos resultados estuvieron entre 50 y 1000 $\mu \mathrm{g} \mathrm{L}^{-1}$, intervalo letal de Cr (LC50-96 h) para Palaemonetes pugio (Doughtie y Ranga, 1984). Además, fueron mucho menores que CCM (1 $100 \mu \mathrm{g}$ $\left.\mathrm{L}^{-1}\right)$ y mayores que $\mathrm{CCC}\left(50 \mu \mathrm{g} \mathrm{L}^{-1}\right)$ de las referencias de la NOOA (Buchman, 2008). Es probable que sus concentraciones estén relacionadas con las características mineralógicas de la zona, de hecho, el cromo total covarió significativamente con arena $(\mathrm{r}=0.63)$. Arauzo et al. (2003) señalan que, en aguas marinas, la movilización del $\mathrm{Cr}$ a la fase líquida está favorecida por la oxidación natural del mismo.

En el saco del golfo de Cariaco, los hidrocarburos aromáticos policíclicos de alta masa molar (HAPA) son dominantes, en especial el fluoranteno. Debido a su alta masa molar, son moléculas hidrofóbicas y lipofílicas, por lo tanto, son poco solubles en agua y es probable que se asienten en el fondo, donde se unen fuertemente a la materia orgánica (Sakari, 2012; Mahugija et al. 2017). Esta primacía junto a los índices fluoranteno/pireno mayor que $1 \mathrm{y}$ fenantreno/antraceno menor que 10 certifican el origen pirogénico de los HAP, tal vez, derivados de la combustión de biomasa, emisiones vehiculares y lavados de las superficies de las carreteras (Men et al. 2009). Estos residuos asociados con partículas entran al aire y luego se redistribuyen en los diferentes compartimientos ambientales (aire, agua, suelo, sedimento), regidos por las propiedades de los 
destinos y las condiciones meteorológicas (Kim et al. 2009; Mahugija et al. 2017). Otros factores pueden ser aguas residuales urbanas, emisiones de motores fuera de borda y descargas directas irresponsables de aceite de motor y gasolina (Terrado et al. 2010). Cabe resaltar que HAPA son considerados trazadores de vehículos a gasolina (Amador-Muñoz et al. 2010).

La concentración de los HAP, en el área de estudio, no superó ERL (Cuadro 4), es decir, no deben provocar efectos adversos en los recursos biológicos, ni en la salud humana. $\mathrm{Su}$ adsorción no estuvo influenciada por el contenido de carbono orgánico, ni por el carácter fino del sedimento (arenas medias, arenas finas y limo). Respecto a esto, algunos investigadores aseguran que su acumulación depende de la cantidad de aportes antrópicos y solamente ocurre en sitios muy contaminados, con más de $2000 \mathrm{ng} \mathrm{g}^{-1}$ (Men et al. 2009).

Como las pruebas de toxicidad con Artemia sp. no presentaron mortalidad de individuos y tampoco hubo evidencia de actividad hemolítica puede decirse que los sedimentos no son peligrosos para los recursos vivos (Chapman, 1996; Long et al. 2002).

Una vez compilados, evaluados y discutidos los resultados de la caracterización química sedimentaria (metales, HAP) y los puntos finales de las pruebas toxicológicas, respecto al control negativo, fueron asignados los valores correspondientes, de acuerdo con los criterios seleccionados (Cuadro 2) y se completó la matriz tabular de decisión para la evaluación semicuantitativa (Cuadro 5), donde se observa que ninguna especie química estudiada excedió el criterio de calidad ERL y las pruebas toxicológicas estuvieron libres de daños o pérdidas; por tanto, califican con puntuación "1"; sin embargo, $\mathrm{Cd}, \mathrm{Cu}$ y $\mathrm{Ni}$, en los sedimentos, fueron categorizados con la puntuación " 2 ", porque presentaron contenidos entre ERL y ERM en $100 \%, 70 \%$ y $42 \%$ del área, respectivamente (Long et al. 2002; Simpson et al. 2005).

Como no ocurrieron efectos toxicológicos adversos, incluyendo agudos y crónicos, ni reducción en los criterios de valoración toxicológicos presagiados por concentraciones químicas elevadas de $\mathrm{Cd}$, $\mathrm{Ni}$ y $\mathrm{Cu}$, se puede conceder a los sedimentos valoración global " 1 " y catalogarse como de Alta Calidad Ambiental, es decir, son inofensivos para los recursos biológicos y la salud humana (Simpson et al. 2005). 


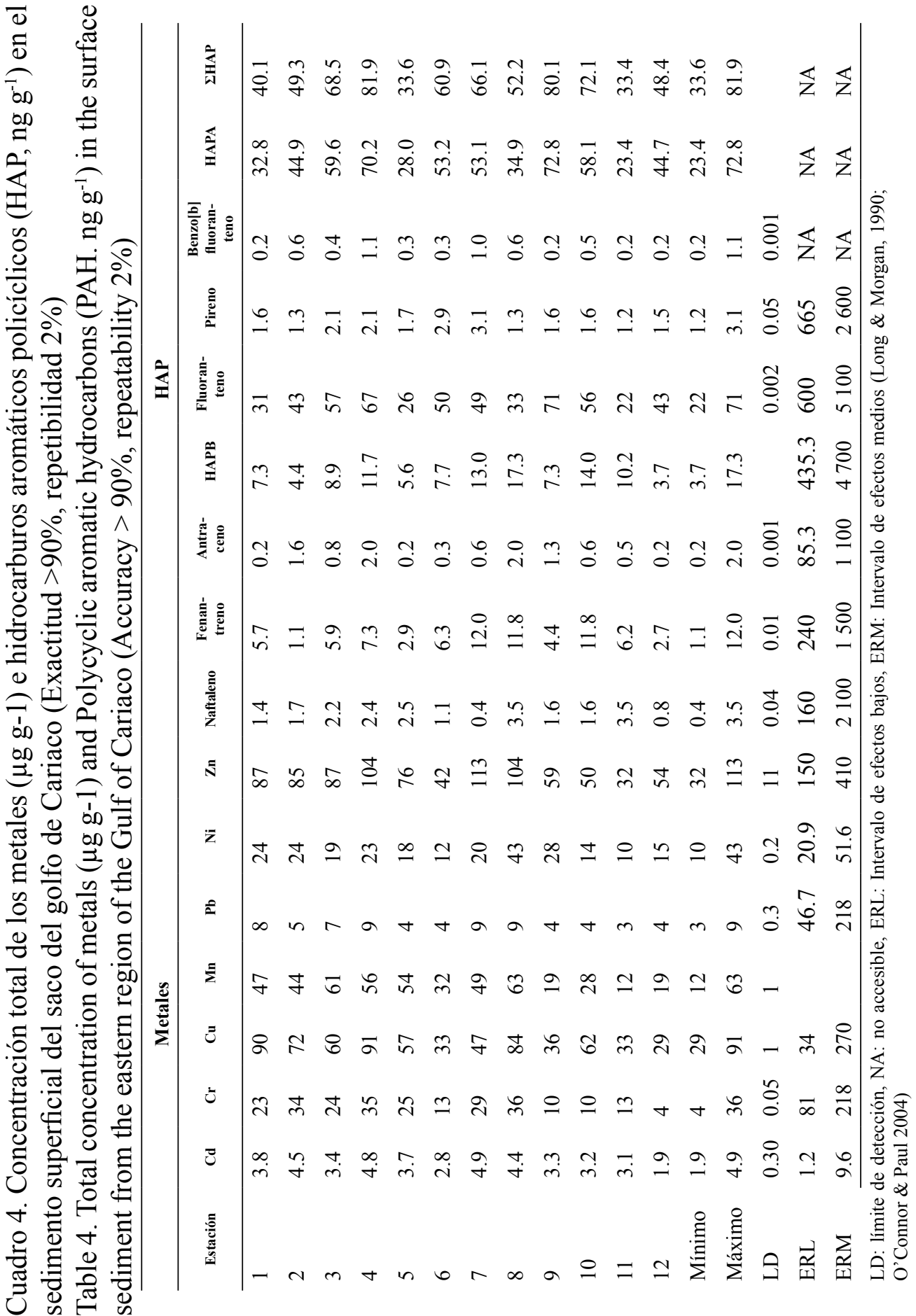


Cuadro 5. Matriz tabular de decisión para la evaluación semicuantitativa de los sedimentos del saco del golfo de Cariaco

Table 5. Tabular decision matrix for the semi-quantitative evaluation of the sediments from the eastern region of the Gulf of Cariaco

\begin{tabular}{llllll}
\hline Estación & $\begin{array}{l}\text { Química } \\
\text { sedimentaria } \\
\text { (metal-HAP) }\end{array}$ & $\begin{array}{l}\text { Química } \\
\text { agua }\end{array}$ & $\begin{array}{l}\text { Supervivencia de } \\
\text { Artemia } \\
\text { (Agua - sedimento) }\end{array}$ & $\begin{array}{l}\text { Actividad } \\
\text { hemolítica }\end{array}$ & Calidad \\
\hline 1 & $2-1$ & $1-1$ & $1-1$ & 1 & Alta \\
2 & $2-1$ & $1-1$ & $1-1$ & 1 & Alta \\
3 & $2-1$ & $1-1$ & $1-1$ & 1 & Alta \\
4 & $2-1$ & $1-1$ & $1-1$ & 1 & Alta \\
5 & $2-1$ & $1-1$ & $1-1$ & 1 & Alta \\
6 & $2-1$ & $1-1$ & $1-1$ & 1 & Alta \\
7 & $2-1$ & $1-1$ & $1-1$ & 1 & Alta \\
8 & $2-1$ & $1-1$ & $1-1$ & 1 & Alta \\
9 & $2-1$ & $1-1$ & $1-1$ & 1 & Alta \\
10 & $2-1$ & $1-1$ & $1-1$ & 1 & Alta \\
11 & $2-1$ & $1-1$ & $1-1$ & 1 & Alta \\
12 & $2-1$ & $1-1$ & $1-1$ & 1 & Alta \\
\hline
\end{tabular}

\section{CONCLUSIONES}

Las concentraciones de los metales y las afinidades entre sí demuestran su procedencia litogénica regional, con muy poca influencia antrópica. De manera similar, la fuente más probable de los hidrocarburos aromáticos policíclicos es la combustión de biomasa y emisiones de vehículos a gasolina. $\mathrm{Su}$ presencia no superó criterios de peligrosidad predicha por concentración química elevada. Por lo tanto, los sedimentos del sector oriental del saco del golfo de Cariaco poseen calidad ecológica alta o buena, donde no hubo evidencias de ocurrencia de efectos adversos, por lo tanto, no representan riesgos para los recursos biológicos, ni riesgos a la salud humana. Esto fue demostrado con los puntos finales negativos de las pruebas toxicológicas.

Este conocimiento de la condición ambiental del saco del golfo de Cariaco permitirá identificar posibles eventos derivadas del acumulo de sustancias químicas naturales y antrópicas que puedan arriesgar la salud del ecosistema y poder administrar el desarrollo económico sostenible de las comunidades costeras aledañas. 


\section{AGRADECIMIENTO}

La autora agradece al Instituto de Investigaciones de Biomedicina y Ciencias Aplicadas (IIBCA-UDO), al Instituto Oceanográfico de Venezuela (IOV-UDO, a los Departamentos de Química de la Universidad de Oriente (UDO) y Universidad Central de Venezuela (UCV) por el apoyo brindado para la realización de este estudio, y a los evaluadores de esta prestigiosa revista por la acuciosa revisión del trabajo.

\section{BIBLIOGRAFÍA}

Ahmad, A., Mushrifah, I. \& Shuhaimi-Otham, M. (2009). Water quality and heavy metal concentrations in sediment of Sungai Kelatan, Kelatan, Malaysia: A baseline study. Sains Malays, 38(4), 435-442.

Amador-Muñoz, O., Villalobos-Pietrini, R., Agapito-Nadales, M., Munive-Colín, Z., Hernández-Mena, L., ... \& Guzmán-Rincón, J. (2010). Solvent extracted organic matter and polycyclic aromatic hydrocarbons distributed in size-segregated airborne particles in a zone of México City: seasonal behaviour and human exposure. Atmos. Environ., 44(1), 122-130.

Araujo, P. A., Shimizu, G., Borher, M. B. \& Jardim, W. (2006). Avaliação da qualidade de sedimentos. En P. A. Zagatto $\&$ E. Bertorelli (Eds.), Ecotoxicologia aquática (pp. 293-326). Brasil.: RiMa Editora.

Arauzo, M., Rivera, M., Valladolid, M., Noreña, C. \& Cedenilla, O. (2003).
Contaminación por cromo en el agua intersticial, en el agua del cauce y en los sedimentos del río Jarama. Limnetica, 22(3-4), 87-100.

Banjoo, D. \& Nelson, K. (2005). Improved ultrasonic extraction procedure for the determination of polycyclic aromatic hydrocarbons in sediments. J. Chromatogr. A., 1066 (1-2), 9-18.

Belzunce-Segarra, M., Prego, R., Wilson, M., Bacon, J. \& Santos-Echeandía, J. (2008). Metal speciation in surface sediments of the Vigo Ria (NW Iberian Peninsula). Sci. Mar., 72(1), 119-126.

Bouyoucos, G. (1962). Hydrometer method improved for making particle size analyses in soils. Agronomy J., 544, 464-465.

Botté, S., Freije, H. \& Marcovecchio, J. (2007). Dissolved heavy metal (Cd, $\mathrm{Pb}, \mathrm{Cr}, \mathrm{Ni}$ ) concentrations in surface water an porewater from Bahía Blanca estuary tidal flats. Bull. Environ. Contam. Tox., 79(4), 415-421.

Buchman, M. F. (2008). NOOA Screening Quick Reference Tables. https://response.restoration.noaa.gov/sites/default/files/SQuiRTs.pdf

Caraballo, L. (1982). El golfo de Cariaco. Parte II. Los sedimentos superficiales y su distribución por el fondo. Fuente de sedimentos. Análisis mineralógico. Bol. Inst. Oceanogr. Venezuela, 21(12), 37-65.

Carballo, H. O., Arencibia, C. G., Concepción, J. \& Isla, M. M. (2010). Los bioensayos de toxicidad en sedimentos marinos. RETEL, 32, 33-69.

Chapman, P. M. (1996). Presentation and interpretation of Sediment Quality Triad data. Ecotoxicology, 5, 327-394.

Doughtie, D. \& Ranga, K. (1984). Histopathological and ultrastructural changes in the antennal gland, midgut, hepatopancreas and gill of grass shrimp 
following exposure to hexavalent chromium. Invertebr. Path., 43(1), 89-108.

Ferráz-Reyes, E. (1987). Productividad primaria del Golfo de Cariaco. Bol. Inst. Oceanogr. Venezuela. Univ. Oriente, 26(1-2), 97-110.

Ferrer, L., Andrade, S., Asteasuain, R. \& Marcovecchio, J. (2006). Acute toxicities of four metals on the early life stages of the crab Chasmagnathus granulata from Bahia Blanca estuary, Argentina. Ecotox. Environ. Saf., 65(2), 209-217.

Fuentes, M. V. (2010). Evaluación de la calidad de los sedimentos superficiales del saco del golfo de Cariaco, Estado Sucre, Venezuela. (Tesis de doctorado no publicada). Universidad de Oriente, Venezuela.

Fuentes, M. V., Rojas, L., Díaz A. \& Martínez, G. (2010). Distribución de metales pesados en los sedimentos superficiales del saco del golfo de Cariaco, Sucre, Venezuela. Rev. Biol. Trop., 58(Suppl. 3), 129-140.

Fuentes, M. V., Daoukdouk S., Rojas, L., Escalona A. \& Ramírez, A. (2012). Presencia y fuentes de hidrocarburos policíclicos aromáticos en los sedimentos superficiales del saco del Golfo de Cariaco. Interciencia, 37(2), 99-106.

Fuentes-Hernández, M. V., Sanguinetti-Gamboa, O. A. \& Rojas-Astudillo. L. L. (2019). Evaluación del riesgo ambiental de metales pesados en los sedimentos superficiales del saco del golfo de Cariaco. Rev. Int. Contam. Ambie., 31(1), 101-114.

García-Rico, L., Soto, M., Jara, M. \& Gómez, A. (2004). Fracciones geoquímicas de $\mathrm{Cd}, \mathrm{Cu} \mathrm{y} \mathrm{Pb}$ en sedimentos costeros superficiales de zonas ostrícolas del Estado de Sonora, México. Rev. Intern. Contam. Ambient., 20(4), 159-167.
Gaudette, H., Flight, W., Toner, L \& Folger, D. (1974). An inexpensive titration method for the determination of organic carbon in recent sediments. J. Sed. Petrol., 44(1), 249-253.

Gutiérrez, J. (2012). La variación y su significado. Rev. Universidad Eafit, 32(101), 87-96.

INE. Instituto Nacional de Estadísticas. (2014). XIV Censo de Población y Vivienda. Resultados por entidad federal y municipio. Caracas, Venezuela.: Instituto Nacional de Estadísticas de la República Bolivariana de Venezuela. http:// www.ine.gov.ve/documentos/ Demografia/Censo de Población y Vivienda/pdf/sucre.pdf.

Key, L., Boyle, P. \& Jaspars, M. (2002). Novel activities of saliva from the octopus Eledone cirrhosa (Molusca, Cephalopoda). Toxicon, 40(6), 677-683.

Khaled, A., Abdel-Halim, A., El-Sherif, Z. \& Mohamed, L. (2017). Health risk assessment of some heavy metals in water and sedimental at Marsa-Matrouh, Mediterranean Sea, Egypt. J. Environ. Prot., 8, 74-97.

Kim, S., Lee, D, Shim, W., Yim, U. \& Shing, Y. (2009). Interrelationship of pyrogenic polycyclic aromatic hydrocarbons (PAH) contamination in different environmental media. Sensors, 9(12), 9582-9602.

Krupadam, R., Smith, P. \& Wate, R. (2006). Geochemical fractionation of heavy metals in sediments of Tapi estuary. Geoch. J., 40(5), 513-522.

Long, E. R. \& Morgan, L. G. (1990). The potential for biological effects of sediment-sorbed contaminants tested in the National and Trends Program. EE. UU.: National Oceanic and Atmospheric Administration (NOOA).

Long, E. R., Dutch, M., Aasen, S., Welch, K., Hameedi, J., Magoon, S... \& 
Anderson, J. (2002). Sediment quality in Pudget Sound Year 3-Sourthern Puget Sound. EE. UU.: National Oceanic and Atmospheric Administration (NOOA).

MacDonald, D., MacDonald, M., O’Brien, M. $\&$ Gobeil, C. (1991). Accumulation of heavy metals $(\mathrm{Pb}, \mathrm{Zn}, \mathrm{Cu}, \mathrm{Pb})$, carbon and nitrogen in sediments from strait of Georgia, B. C. Canada. Mar. Chem., 34(1-2), 109-135.

Mahugija, J., Ahmed, K. \& Makame, Y. (2017). Polyciclic aromatic hydrocarbons (PAHs) contamination in coastal mangrove ecosystems of the Zanzibar archipielago. WIO J. Mar. Sci., 16(1), 25-34.

Man, K., Zheng, J., Leung, A., Lam, P., HonWah, M. \& Yen, F. (2004). Distribution and behavior of trace metals in the sediment and porewater of a tropical coastal wetland. Sci. Tot. Environ., 32(7), 295-314.

Márquez, A., Bonilla, J., Martínez, G., Aguilera, D. \& González, A. (2005). Estudio geoquímico de los sedimentos superficiales del litoral nororiental del golfo de Cariaco, Estado Sucre, Venezuela. Bol. Inst. Oceanogr. Venezuela. Univ. Oriente, 44(2), 89-103.

Martínez, G. (2002). Metales pesados en los sedimentos superficiales del golfo de Cariaco, Venezuela. Bol. Inst. Oceanogr. Venezuela, 41(1-2), 83-96.

Martínez, G. (2016). Calidad Ambiental de los sedimentos recientes del golfo de $\mathrm{Ca}$ riaco. Trabajo de ascenso no publicado, Universidad de Oriente, Venezuela.

Men, B., He, M., Tan, L., Lin, C. \& Quan, X. (2009). Distributions of polycyclic hydrocarbons in the Daliao river estuary of Liaodong, Bohai Sea (China). Mar. Poll. Bull., 58(6), 818-826.

Nayar, S., Goh, B. \& Chou, L. (2004). Environmental impact of heavy metals from dredged and resuspended sediments on phytoplankton and bacteria assessed in situ mesocosms. Ecotoxicol. Environ. Safe., 59(3), 349-369.

NOOA. National Oceanic and Atmospheric Administration. (2019). Screening Quick Reference Tables. EE. UU.: Office of Response and Restoration Division, National Oceanic and Atmospheric Administration.

O’Connor, T. P. \& Paul, J. F. (2004). The Sediment Quality Guideline, ERL, is not a chemical concentration at the threshold of sediment toxicity. Mar. Poll. Bull., 49(5-6), 383-385.

Primo, E. \& Carrasco, J. (1973). Química Agrícola I. Suelos y fertilizantes. España.: Editorial Alhambra, S. A.

Quintero, A., Terejova, G., Vincent G., Padrón A. \& Bonilla, J. (2002). Las corrientes marinas en el golfo de Cariaco, Venezuela. Bol. Inst. Oceanogr. Venezuela, Univ. Oriente, 48(2), 109-119.

Quintero, A., Terejova, G. \& Bonilla, J. (2005). Morfología costera del golfo de Cariaco, Venezuela. Bol. Inst. Oceanogr. Venezuela, Univ. Oriente, 44(2), 133-143.

Quintero, A., Caraballo, L., Bonilla, J., Terejova, G. \& Rivadula, R. (2006). Sedimentos marino-costeros del golfo de Cariaco, Venezuela. Bol. Inst. Oceanogr. Venezuela, Univ. Oriente, 45(2), 127-139.

Rojas, L., Chang-Yen, I. \& Bekele, I. (2005). Heavy metals in sediments, mussels and oysters from Trinidad and Venezuela. Rev. Biol. Trop., 53(1), 33-40.

Rubio, B., Nombelas, M., Vilas, F., Alejo, J., García-Gil, S., García-Gil, E. \& Pazos, O. (1995). Distribución de metales pesados en sedimentos actuales de la parte interna de la ría de Pontevedra. Thalassas, 11, 35-45.

Sakari, M. (2012). Depositional history of polycyclic aromatic hydrocarbons: 
reconstruction of petroleum pollution records in Peninsular Malaysia. In T. Puzym \& A. Mostrag (Eds.), Organic pollutants ten years after Stockholm Convention-environmental and analytical update (pp. 135-165), UK.: Intech Open Access Publisher.

Salazar, S. (1991). Contaminación marina. México.: Fondo de Publicaciones y Ediciones del Centro de Investigaciones de Quintana Roo.

SCFI. Secretaría de Comercio y Fomento Industrial. (1995). (NMX-AA-110-SCFI-1995). Análisis de agua. Evaluación de toxicidad aguda con Artemia franciscana Kellogg (Crustacea - Anostraca)-Método de prueba. México.: Dirección General de Normas.

Shepard, F. (1954). Nomenclature based on sand-silt-clay ratio. $\mathrm{J}$. Sed. $\mathrm{Pe}$ trol. 24, 151-158. https://doi.org/10.1111/j.1365-3091.1966.tb01598.x

Simpson, S., Batley, G., Chariton, A., Stauber, J., King, C., Chapman, J., ... \& Maher,
W. (2005). Handbook for sediment quality assessment. Australia: CSIRO.

Swarnalatha, K. \& Nair, A. (2017). Assessment of sediment quality of a tropical lake using sediment quality standards. Lakes \& Reservoirs, 22(1), 65-73.

Terrado, M., Barceló, D. \& Tauler, R. (2010). Multivariate curve resolution of organic pollution patterns in Ebro River surface water-groundwater-sedimentsoils system. Anal. Chim. Acta, 657(1), 19-27.

Velásquez, A. (2005). Distribución y comportamiento de los metales pesados $\mathrm{Cd}$, $\mathrm{Cu}, \mathrm{Ni}, \mathrm{Zn}, \mathrm{Fe}, \mathrm{Mn}, \mathrm{Co}, \mathrm{Cr}$ y $\mathrm{Pb}$ en los sedimentos superficiales del sector oriental del golfo de Cariaco. Estado Sucre. (Tesis Pregrado no publicada). Universidad de Oriente, Venezuela. 